\title{
NAMEN ALS JÜDISCHE IDENTITÄTSATTRIBUTE UND ALS MitTel Des HuMORS ODER DER VERSPOTTUNG IN DEN WITZBLÄTTERN (1882-1883)
}

\author{
Ágnes TAMÁs \\ Universität Szeged, Hungary

\begin{abstract}
Names as attributes of Jewish identity and tools of humour and irony in humour magazines (1883-1883)
\end{abstract}

\begin{abstract}
The paper aims to analyse names that appeared in caricatures and texts of humour magazines (Borsszem Jankó, Bolond Istók, Üstökös) published in 1882-1883, after the Tiszaeszlár Affair. The author analyses the names of Israelite characters, discusses how the magazines comment on name changes, and analyses fictitious toponyms and the nicknames of individuals who may be linked to the Nyíregyháza case.

The magazine issues examined are not of interest because the adoption of Hungarian family names is a frequent topic in them, but mostly because names are the primary source of humour and irony.

Keywords: humour magazines, Jewish family names, name change, fictitious toponyms.
\end{abstract}

\section{Einleitung}

Im Zeitalter des Dualismus können die Jahre 1882 und 1883 als Höhepunkt des Antisemitismus im 19. Jahrhundert in Ungarn betrachtet werden. Das Korpus der Analyse, die Witzblätter mit Karikaturen waren - nicht nur in Ungarn - populär in der zweiten Hälfte des 19. Jahrhunderts. Die ausgewählten Jahrgänge der Witzblätter erschienen während des Nyíregyházer Prozesses der ungarischen Juden mit der Anklage eines Ritualmordes. Der angebliche Tiszaeszlárer Ritualmord (im April 1882), der Prozess der Juden in Nyíregyháza (1883) und die antisemitischen Bewegungen des Jahres 1883 interessierten die Zeitgenossen äußerst. Die Nachrichten über den Ritualmord verbreiteten sich schnell sowohl in der ungarischen als auch in der europäischen Presse. Die antisemitische Presse stellte die Geschichte folgenderweise dar: Am 1. April 1882 verschwand ein Dienstmädchen (Eszter Solymosi) in Nordostungarn, in Tiszaeszlár, wo sich die Juden versammelten um Passah zu feiern. Laut der Anklage entnahmen die Juden - in erster Linie Salamon Schwarz - das Blut des Dienstmädchens um Osterbrot zu backen. Die Untersuchung und der Prozess der Juden erfolgte in Nyíregyháza, in der Nähe von Tiszaeszlár und dauerten lange, bis zum August 1883, aber die antisemitische Welle war nicht zu Ende: In Ungarn 
begannen antisemitische Hetzkampagnen gegen die Juden, die monatelang dauerten. Die Israeliten wurden geschlagen, beraubt, die Fenster ihrer Geschäfte wurden eingeschlagen (siehe Gottas 1976: 168-179, Fischer 1988: 42-46, 77-82). Es ist wertvoll, die Presseorgane dieser konfliktreichen Periode zu analysieren.

In dieser Studie untersuche ich das Namenmaterial eines semitischen Witzblattes (Borsszem Jankó [Budapest]) und zwei antisemitischer Witzblätter (Üstökös und Bolond Istók [Budapest]) von dem ersten Erscheinen des Themas bis zum Ende des Jahres 1883. Borsszem Jankó probierte seine Leserschaft davon zu überzeugen, dass die Klagen gegen die Juden ganz absurd sind, dagegen agitierten Bolond Istók und Üstökös gegen die Juden. Der Editor des liberalen, regierungsfreundlichen Witzblattes, Borsszem Jankó war ein jüdischer Arzt, Adolf Ágai und viele von seinen Mitarbeitern waren auch Israeliten. Bolond Istók und Üstökös können als oppositionelle Presseorgane betrachtet werden.

Durch die Personennamen konnte man die Figuren der Witzblätter rapid identifizieren, oder den Figuren neue Identitäten mit Hilfe von Personennamen geben. Die Namen der Angeklagten und Advokaten (Károly Eötvös, Nándor Horánszky, Sándor Funták, Bernát Friedmann und Miksa Székely; Anklagevertreter: Ede Szeyffert) wurden schnell im ganzen Land bekannt. Eötvös war ein berühmter Jurist seiner Zeit, hatte keine jüdischen Vorfahren, und viele seiner Kollegen ermahnten ihn, dass er diese Rechtssache nicht übernehmen darf, weil dieser Prozess das Ende seiner Karriere bedeuten kann. Die Namen der Hauptpersonen des Prozesses wurden ausgespottet, was ich darlege, sowie die Verspottung des Magyarisierungsprozesses der Namen. In den Witzblättern war es untypisch, dass die Namen die Hauptrolle in Karikaturen spielten. Aus diesen Jahrgängen präsentiere ich trotzdem solche Karikaturen. Diese enthalten erfundene Toponyme (ausgespottete jüdische Familiennamen lebendiger Personen oder andere Stereotype über Juden als fiktive Toponyme) und Personennamen im stereotypen, antisemitischen Kontext. Erfundene Toponyme können auch nicht als typischer Inhalt der Witzblätter betrachtet werden.

\section{Stereotype jüdische Familiennamen der Witzblätter}

Vor der Analyse der Namen, die irgendwie im Zusammenhang mit dem Ritualmord oder mit dem Prozess stehen, präsentiere ich die folgende Karikatur, die die zeitgenössischen Vorurteile und Stereotype über die Juden zusammenfasst und zeigt, wie leicht einfach und verständlich diese Präjudize mit Familiennamen auf einer Karikatur dargelegt werden können. Die Familiennamen oder die Elemente der Namen sind für typisch jüdisch gehaltene Personenamen der Epoche. Der Karikaturist erreichte die humoristisch-sarkastische Wirkung so, dass die Bedeutung der Namen gegensätzlich mit der Aussicht der Figuren ist, aber alle Charaktere sehen widerlich aus. Die Figur mit dem Namen Gross ist sehr klein, dagegen ist der Jude Klein sehr hoch und übergewichtig. Weiss hat schwarzes Gesicht, Dick ist so dünn, dass er fast nicht stehen kann, Spitz hat dagegen Mehrgewicht. Die Figur Ehrlich besticht jemanden und Löw ist nicht so brav wie ein Löwe, weil er vor einer Maus erschrak. Rosenblüh stinkt 
sehr, was die zeitgenössischen Stereotype wiederspiegelt: Die Antisemiten erwähnten oft, dass die Juden fast nicht baden und viel Knoblauch essen, deswegen stinken sie. Tänzer hat X-Beine, Schön sieht nicht so ästhetisch aus, Gutherz ist reich, aber er vertreibt einen jüdischen Bettler (Üstökös, 9. Dezember 1883).

Die Anzahl der Personennamen ist geringer in diesen zwei Jahrgängen der Witzblätter und die Varietät der Elemente der Familiennamen ist auch nicht so hoch, und es ist augenfällig, dass die übrigens häufigen Spitznamen der Witzblätter nicht so oft vorkommen (siehe Tamás 2015: 370-371). Eine Neuigkeit kann doch in einem der antisemitischen Witzblätter wahrgenommen werden. Der zeitgenössische Autor typisierte, gruppierte die jüdischen Familiennamen in einem längeren Text. Am Anfang des Dialoges stellte er fest, dass er sich mit dem jüdischen Personennamen nicht befreunden kann, und er erzählt über die Namengebungsbräuche der ungarischen Juden. Anhand des Textes gibt es Juden, die „geographischen“ oder „naturkundlichen“ Namen haben, und auch solche, die „historischen“ Familiennamen besitzen. Die „geographischen Juden“ haben noch unterschiedliche Untergruppen: Sie bekamen Ländernamen oder Namen von Städte wie zum Beispiel Friedländer, Holländer oder Hamburger. Die "naturkundlichen Juden“ besitzen Namen aus der Tierwelt (z. B.: Löw, Fuchs) inklusive Menschen (z. B.: Neumann), aus der Pflanzenwelt (z. B.: Grünbaum) und von Mineralien und Edelsteinen (z. B.: Diamantstein, Goldstein). Er erklärt die historischen Namen nicht, weil der zeitgenössische Leser wusste, dass er an die Juden denkt, die ihre Namen so magyarisierten, dass sie die Familiennamen von ungarischen historischen Personen (z. B.: Zrínyi, Rákóczi) aufnahmen (Bolond Istók, 29. Juli 1883). Diese letzte Variante der Magyarisierung der jüdischen Familiennamen wurde heftig kritisiert. Wenn man unter die Kategorie „Menschen“ auch die Namen, die innere oder äußere Eigenschaften sowie körperliche Behinderungen beinhalten, versteht, kann behauptet werden, dass diese Typologie fast die Gesamtheit der Familiennamentypen der Witzblätter darstellt (Tamás 2015: 370-371).

Die Erklärung dafür, dass das Personennamenmaterial nicht so abwechslungsreich ist wie in anderen Jahrgängen derWitzblätter, kann sein, dass die meisten jüdischen Personennamen irgendwie mit dem Ritualmord und dem Prozess zusammenhängen.

\section{Spitznamen der Personen des Prozesses}

Die antisemitischen Witzblätter spotteten die Namen der jüdischen Angeklagten und ihrer Advokaten aus, indem Borsszem Jankó die Namen der antisemitischen Politiker lächerlich machte. Gyula Verhovay, der den angeblichen Ritualmord in seiner Zeitung mit dem Titel Függetlenség ('Unabhängigkeit') zuerst veröffentlichte, und später einer der Gründer der Antisemitischen Partei Ungarns wurde, kommt als Vérhasovay vor. Sein Spitzname klingt Verhovay ähnlich und bedeutet Blut ('vér') und Magen ('has'). Das -y am Ende suggeriert seine adelige Abstammung. Sein erfundener Name, der in Borsszem Jankó sehr oft benutzt wurde, deutet auf Blutrausch hin, und zeigt wie Verhovay die Judenfrage nach der Meinung von Borsszem Jankó - nicht 
friedlich, sondern blutig - lösen will. Er spricht in den Texten der humoristischen Zeitung immer wieder über den Ritualmord, über Blut und blutdürstige Juden.

Die antisemitischen Organe veränderten den Namen von Eötvös, dessen Familienname 'Goldschmied' bedeutet, folgenderweise: Sein Name wurde auf Goldschmied verdeutscht oder er kommt als Aranymives (ein Synonym für Goldschmied auf Ungarisch) vor. Diese zwei Namenvarianten repräsentierten meistens jüdische Figuren in den Witzblättern, also knüpften die Autoren der antisemitischen Texte Eötvös und die Juden ausschließlich durch die Veränderung seines Familiennamens zusammen. Für die Darstellung seiner negativ beurteilten Metamorphose - er wurde visuell auch als Jude abgebildet - war genug die Veränderung des Familiennamens. Wenn man nichts über den Prozess gehört hätte, hätte man wissen können, dass Eötvös irgendein Geschäft mit Juden hatte. Es kommt auch eine einzelartige Namenschöpfung, die in den Witzblättern untypisch war, vor: Eötvös erscheint auch als 5vös. 'Öt' heißt 'fünf' auf Ungarisch (am Anfang des Namens stehende „eö“ ist eine alte Schreibweise des Vokals „ö“), deshalb entsteht das Wortspiel.

Der öffentliche Ankläger (Ede Szeyffert) wurde auch durch die Veränderung seines Namens kritisiert. Während der Prozessverhandlung schien so, dass er lieber mit der Verteidigung einverstanden ist, also kann er seine Arbeit als Ankläger nicht versorgen. Wegen des ähnlichen Anklangs wurde sein Name auf Saupferd verdeutscht. Man kann auch eine Karikatur mit diesem Namen finden: Szeyffert kommt als Tier vor, er besteht aus dem Kopf einer Sau und dem Körper eines Pferdes, auf seinem Rücken reiten die Verteidiger der Juden. Das Wortspiel auf Ungarisch ist deswegen noch interessanter, weil das Wort für „Ankläger" ( vádló") ähnlich dem Wort „wildes Pferd“ ('vad ló') klingt (Bolond Istók, 1. Juli 1883).

Die Autoren der Zeitung Üstökös umgestalteten auch die Namen der Advokaten und des Richters (Ferenc Kornis) folgenderweise: Eötvös Károly > Goldschmied Chaim, Székely Miksa > Michel Czekelesz, Heumann Ignác > Sakter Salabakter, Friedmann Bernát > Beerele Kriegsmann, Korniss > Górnischt („gór“ heißt auf Hebräisch „kein Jude“). Die Autoren benutzten entweder den ähnlichen Klang, oder die Bedeutung der Name, um humoristische Wirkung zu erreichen.

Der jüdische Chefredakteur von Pester Lloyd, von einer deutschsprachigen Tageszeitung (herausgegeben in Budapest), Miksa Falk (Max Falk), der gegen den Ritualmord protestierte, magyarisierte seinen Namen mit der folgenden Argumentation anhand des Bolond Istók: Da der Name Ester ein jüdischer Rufname ist, muss der Name von Eszter Solymosi („sólyom“ auf Deutsch heißt 'Falke') auch ein magyarisierter Name sein. Deswegen magyarisiert Max Falk seinen Namen sofort auf Miksa Sólymossy. Da Eszter Solymosi eine Jüdin ist, und die Juden würden ein jüdisches Mädchen nie töten, können die Juden von Tiszaeszlár nicht schuldig sein (Bolond Istók, 25. Jun. 1882). Die humoristische Falschmeldung der antisemitischen Zeitung benutzte die Bedeutung der Familiennamen als Mittel um humoristische Effekte zu wecken. Solches Mittel wurde auch auf einer Karikatur verwendet. Mór Lichtmann war ein Zeuge des Prozesses, und er kommt in einem dunklen 
Hintergrund vor, dagegen erscheint Schwarz auf einem weißen Hintergrund (Üstökös, 15. Juli 1883).

\section{Magyarisierung der Familiennamen}

Während einerseits sich die antisemitische Gesinnung in diesen Jahren ausbreitete, andererseits die Anzahl der akzeptierten Eingaben der Juden mit dem Wunsch der Namenmagyarisierung an das Innenministerium auch sehr hoch war (teilweise wegen des Wachstums des Antisemitismus) (Karády und Kozma 2002: 60-62). Die zahlreichen Namenmagyarisierungen waren den Zeitgenossen auffällig, deswegen beschäftigten sich die Witzblättereditoren damit öfter in diesen Jahren. Das Interesse für das Thema war hoch, was zeigt, dass das Regierungspropaganda die Magyarisierung der fremden Familiennamen drängte (siehe Maitz 2009). Die Antisemiten empfingen aber diese symbolischen Akte (Magyarisierung der Namen und Schmad) mit Widerwille, sie protestierten gegen die Aufnahme von historischen ungarischen Namen und Namen von Adelsfamilien. Die antisemitischen Texte akzentuierten, dass wenn der Jude ungarischen Namen wählt, daraus folgt noch nicht, dass er Ungar sein wird, er bleibt davon unabhängig ein Jude.

Eine Karikatur zeigt diese Erscheinung und die Leidenschaft der Zeitgenossen: Miklós Zrinyi („origineller“ jüdischer Name: Mendl Zieringer) rennt aus seinem Geschäft in Szigetvár aus, und er flüchtet sich vor der antisemitischen Hetze gegen ihn, gegen die Juden (Borsszem Jankó, 9. September 1883). Zrínyi ist ein historischer Familienname von berühmter Adelsfamilie, deren Mitglieder gegen die Türken kämpften. Miklós Zrínyi verteidigte die Burg in Szigetvár einen Monat lang gegen die riesengroße osmanische Armee im Jahre 1566, und starb den Heldentod. Die Karikatur setzte den "neuen“ Zrinyi mit dem „alten" entgegen: Der neue hat Angst vor ein paar Magyaren, der alte kämpfte gegen ein Heer mit ca. 50.000 Soldaten. Oder eine andere Interpretation kann auch möglich sein: Die Hetzkampagnen, die Antisemiten sind genauso gefährlich wie die osmanische Armee. In den 1880er Jahren erlaubten die Gesetze, dass man Namen von adeligen, alten Familien aufnimmt, man konnte den magyarisierten Namen fast frei auswählen, man sollte nur die altmodische Schreibweise (Benutzung von -y, -eö, -ch, -tt) vermeiden (Karády und Kozma 2002: 60-61), dennoch wollten viele Personen Familiennamen mit traditioneller Schreibweise wählen (Farkas 2012: 304). Im Interesse des Prestiges und der Verteidigung der Familiennamen formulierten die Zeitgenossen ihren Anspruch auf eine detaillierte Regelung der Möglichkeiten der Namenmagyarisierung (Nagy 1992-1993: 67), aber diese Maßregeln wurden nur viel später, im Jahre 1933 gebracht (Farkas 2002: 160). Auf einer anderen Karikatur sieht man die Geschäfte der Juden (Iczik Rákóczy, Jakab Batthiány, Izidor Frangepán), die während katholischen Feiertagen geöffnet, während jüdischen Festen geschlossen sind (Bolond Istók, 11. September 1883), und - laut Bolond Istók - es verursacht die schlechte Moral der Magyaren. Alle drei Adelsfamilien spielten wichtige Rolle in der ungarischen Geschichte: Ferenc Rákóczi führte einen Freiheitskampf gegen die Habsburger am Anfang des 18. Jahrhunderts, 
Lajos Batthyány war der Ministerpräsident der ersten ungarischen unabhängigen konstitutionellen Regierung und die Mitglieder der Familie Frangepán kämpften sowohl gegen die Osmanen als auch später gegen die Habsburger. Es kann festgestellt werden, dass die Witzblätterautoren die Familiennamen der Aderligen nicht immer - orthographisch gesehen - richtig benutzten, sie schrieben die Namen mehrmals mit dem -y als das -y wirklich vorkam. Nach dem erfundenen „Gesetzvorschlag" von Bolond Istók sollte man an alle jüdischen Familiennamen den Buchstaben -y ankleben, und -y sollte anstatt des -a der erste Buchstabe des Alphabetes sein (Bolond Istók, 20. August 1882). Um die Kritik der Aufnahme der Namen mit traditioneller Schreibweise zu akzentuieren, wurde -y im Falle von magyarisierten Familiennamen oft in den antisemitischen Witzblättern verwendet.

Es kommen auf Karikaturen auch magyarisierte Spitznamen vor. Im Schaufenster kann man die Namen der Eigentümer der Geschäfte lesen: Körülbüdös Izsák und Ócsó Jakab. Beide Familiennamen enthalten Stereotype. Körülbüdös ('rundherum stinkend') weist auf die zeitgenössische Vermutung, dass die Juden nicht/wenig baden und deswegen sind sie stinkend. Ócsó ('billig', richtig auf Ungarisch: olcsó) deutet vielleicht darauf an, dass der jüdische Schneider nicht so sehr billig ist (Üstökös, 3. September 1882). In den Texten kann man noch andere stereotype magyarisierte Familiennamen lesen, die meistens das Reichtum der Juden suggerierten: Arany ('Gold'), Drágai ('Teurer'), Ezüstkövy ('Silbersteiner'). Die Autoren teilten nicht oft die „originalen“ Familiennamen mit, also über die Motivation der Namenmagyarisierung anhand der Witzblätter ist es wenig zu sagen. Wenn die ungarische und die deutsche Variante zusammen vorkommt, war die Spiegelübersetzung typisch (Steinburg > Kövári ['Stein + Burg + er'], Rosenberg > Rózsahegyi ['Rose + Berg + er']), oder der neue ungarische Familienname begann mit dem gleichen Buchstaben wie der deutsche Name (Kohn > Kún).

\section{Fiktive Toponyme in den Witzblättern}

In dieser Gruppe der fiktiven Namen findet man verändert teils die Namen der Figuren des Tiszaeszlarer Ritualmordes, teils Namen lebendiger, wohlbekannter jüdischer Zeitgenossen. Die Texte und Karikaturen, die erfundenen Namen enthalten, zeigen auch Stereotype über die Juden. Mit den Ereignissen des Prozesses in Nyíregyháza ist die nächste Karikatur eng verbunden. Der Titel, $A z$ új földesúr ('Der neue Grundherr' ${ }^{`}$ weist auf den Roman von Mór Jókai ${ }^{1}$ mit identischem Titel hin. Jókai schilderte in seinem Roman wie sich ein österreichischer Grundherr (Ankerschmidt) in Ungarn assimilierte. Auf der satirischen Zeichnung ist Károly Eötvös auf seinem Grundstück in Mezőszentgyörgy zu sehen (Bolond Istók, 16. September 1883). Eötvös erscheint als Jude, also gelang ihm die Assimilation nicht, die Situation ist gerade umgekehrt wie im Roman von Jókai, weil er sich an die Juden assimilierte. Der Advokat trägt

1 Mór Jókai (1828-1904): Berühmter Autor, Zeitungseditor und Politiker der Epoche, der mehr als hundert Romane und Dramen schrieb. 
jüdische Kleidung, hat Peies, und das Dach des Hauses wurde aus Schächtermessern geformt. Daneben treibt eine jüdische Figur Schafböcke (auf Ungarisch: „kos") auf der Straße mit dem Namen Kos ut ('Kos Straße'). Der Name der Straße ist ein Wortspiel mit dem Namen von Lajos Kossuth. Der berühmte ungarische Politiker, der dieser Zeit im Exil lebte, protestierte heftig gegen den Antisemitismus und gegen den Prozess von Nyíregyháza (z. B.: Kossuth 1904: 117-118). Es ist auch kein Zufall, dass der Jude Schafböcke treibt. Die antisemitischen Zeitgenossen erwähnten oft, dass die Juden Widderkopf haben, was sehr pejorativ verstanden war und kräftig karikiert wurde.

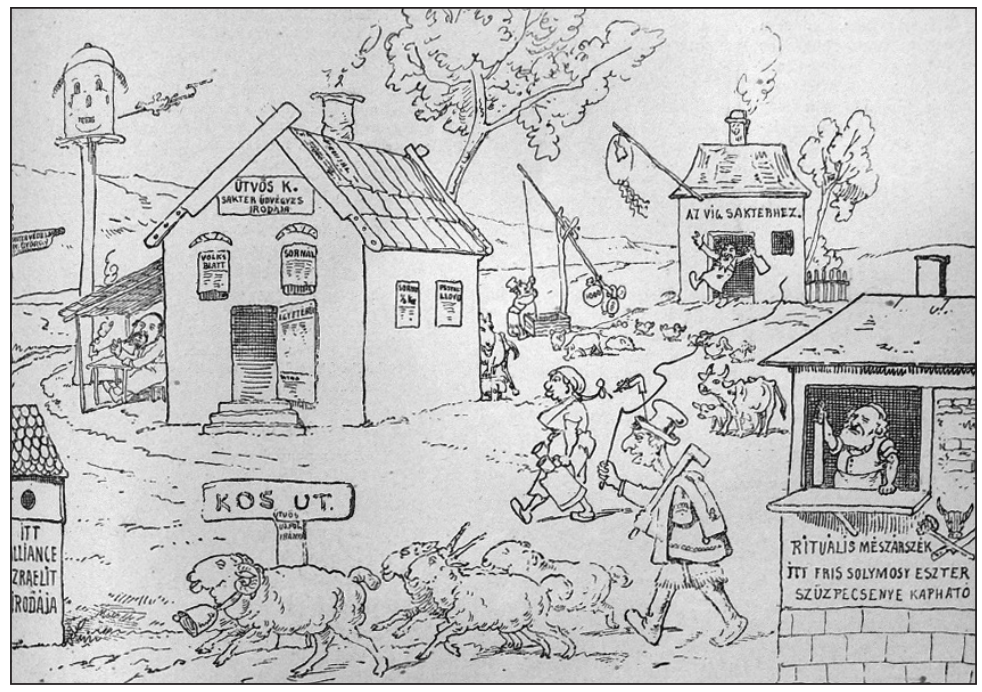

Figur 1. „Der neue Grundherr“

Das Motiv des Schächtermessers kehrte oft in den antisemitischen Zeitungen zurück. Das kann man auch im Wappen von Új Jeruzsálem ('Neu Jerusalem') auf einer Karikatur erblicken (Bolond Istók, 6. August 1882). Auf das Messer wurde der blutige, abgeschnittene Kopf eines Mädchens gezeichnet, also stehet das Bild im engen Zusammenhang mit dem angeblichen Ritualmord von Tiszaeszlár. Die Basis der Karikatur ist die Karte von Budapest, die Skizze ähnelt in großem Maße dem zeitgenössischen Stadtplan von Budapest. Die Toponyme wurden aber modifiziert, Namen als Mittel des Humors spielen Schlüsselrolle auf der Karikatur. Mit der Karikatur wurde die jüdische Ausdehnung in der ungarischen Hauptstadt symbolisch dargestellt und die Kritik der Antisemiten verfasst. Die Anzahl der jüdischen Bevölkerung von Budapest stieg schnell an, in den 1840er Jahren blieb ihr Anteil unter 10\%, um die Jahrhundertwende erhöhte er sich auf mehr als 20\% der Gesamtbevölkerung von Budapest. Um die Relevanz des neuen Stadtnamens zu erhöhen, benannten die Autoren eine der Bergen in Buda (Ofen) als Sion-hegy (Berg Zion wie in Jerusalem). Ein Teil der neuen Straßennamen und die Namen der Stadtteile stammen von 
männlichen und weiblichen für Juden typisch gehaltenen Rufnamen: Rüfke, Kóbi, Iczig, Mojzes, Áron, Salamon, Jajtelesz, Jókelesz, Pinkelesz. Die häufigsten Rufnamen in den Jahren 1882-1883 waren die Folgenden: männlich: Iczig/Izsák ('Isaak'), Jakab/ Kóbi ('Jakob'), Salamon ('Salomon') und Izidor ('Isidor'), jedoch der häufigste weibliche Name (Rozália/Záli) kommt hier nicht vor. Rüfke - in den Witzblättern immer als Name jüdischer Frauen - hatte aber eine pejorative Bedeutung und funktionierte nicht nur als Frauenname. Man nannte die Frauen mit lockerer Moral oder die Prostituierten als „rüfke“. Juden besaßen Lusthäuser in der Innenstadt, sehr viele in dem Stadtviertel, der als Rüfkestadt auf der Karikatur genannt wurde (vgl. Császtvay 2009: 236-258). Die ungarischen Bürger protestierten heftig gegen die Bordelle und gegen die immer lockerere Moral in Budapest (aber benutzten viele von ihnen diese Dienstleistung), und gaben den Juden Schuld an den moralischen Verfall in Budapest. Die meisten Rufnamen haben biblischer Herkunft und kamen im ganzen analysierten Korpus häufig vor.

Eine andere Gruppe der Namen bilden die Familiennamen lebendiger jüdischer Personen als neue Toponyme: Wodjaner utca ('Wodjaner Straße'), Chorin und Rothschild tér ('Chorin und Rothschild Platz'), Szt. Gelléri Mór hegy ('St. Gelléri Mór Berg' in der Realität: Gellérthegy, auf Deutsch: Blocksberg in Ofen). Im letzten Fall ist die Motivation der Namenswahl eindeutig: Der Familienname von Gelléri klingt ähnlich dem Namen Gellért. Mór Gelléri war ein bekannter Publizist, Freimaurer, Mór Wodianer, Ferenc Chorin und die Rothschilds waren alle einflussreiche Geschäftsmänner.

Der Berufsname Schächter kam auch als Bestandteil fiktiver Namen vor: Sakter tér ('Schächter Platz'), Sakter körút ('Schächter Ringstraße') und Közsakterozó híd ('Schlachthaus Brücke'). Nicht nur die Schächter, sondern auch Eszter und - in modifizierter Form - Eszlár (oft benutzte Abkürzung von Tiszaeszlár), und die anderen Personen des Prozesses erschienen auch auf der Mappe. Man kann die Eszter híd ('Ester Brücke'; heute: Margit-híd) und Esler sziget ('Esler Insel'; heute: Margitsziget) anschauen. In beiden Fällen enthält der nicht fiktive Name den ungarischen Frauennamen „Margit“" (auf Deutsch: Margareta). Auf der Esler Insel befindet sich das rituelle Badehaus (Rituális fürdó), daneben die Rabbi sziget ('Rabbiner Insel'), worauf zwei Kaps sind zu sehen: Cap Funták und Cap Horánszky. Beide Herren, Sándor Funták und Nándor Horánszky waren Verteidiger der Juden im Prozess von Nyíregyháza. Nicht mehr an Personennamen, sondern an die Essgewohnheiten der Juden knüpfen sich die nächsten fiktiven Toponyme: Maczesz utca ('Matze Straße'), Disznó tér ('Schweineplatz') in der Nähe des Schlachthauses, Liba hegy ('Gans Berg') und Libamáj utca ('Gänseleber Straße'). Alle Namen weisen darauf hin, dass die Juden traditionell kein Schweinefleisch, sondern lieber Gänsefleisch aßen und wurde auch das jüdische Osterbrot (Matze) erwähnt.

Die Motivation bei den folgenden Namen war vermutlich der ähnliche Klang. Man kann auf der Czepel sziget (in der Realität: Csepel-sziget, Eugensinsel) einen jüdischen Hausierer sehen. Das Wort czepel ist eine Alternativform für cipel auf Ungarisch, 
das auf Deutsch 'schleppen' heißt. Nyuzoda steht für uszoda ('Schwimmbad'), aber das ungarische Verb nyúz bedeutet 'häuten', also ist Nyuzoda eine solche Anstalt, wo man häutet, was auch auf den angeblichen Ritualmord hinweist. Anstatt Császár Fürdő (Kaiser Bad) kann man Csiszár fürdö, und anstatt Múzeum ('Museum') Mojzeum lesen. Csiszár ist ein Name für zwei Berufe, die für Juden für typisch gehalten wurden: einerseits Pferdemakler, andererseits Weinhändler. Noch weitere Stereotype und Vorurteile kommen vor, die an die wirtschaftlichen Tätigkeiten der Juden und an den immer wieder behandelten, angeblichen Betrug der Juden andeutet. Es gibt auf dem Stadtplan einen Fö Rebach tér (heute: Fővámház tér, 'Hauptzollhausplatz', fö [ung. 'haupt'] + Rebach [hebräisch: 'Profit']), Hunczutka utca ('Verschmitzte Straße'), Zsebmetszo" utca ('Taschendieb Straße') und Svindler utca ('Schwindler Straße'). Die Alliancz híd ('Alliancz Brücke'; heute: Lánchíd, Kettenbrücke) beinhaltet teilweise den Namen einer jüdischen Organisation (Alliance Israélite Universelle), die den Juden weltweit materiell und mit Hilfe von Schulung und anderen Arten von Förderung half (Ujvári 1929: 20). Die Antisemiten sahen darin aber eine universale Konspiration der Juden um die Weltmacht zu ergreifen.

Tabelle 1. Zusammenfassung der Namen der Karte

\begin{tabular}{|l|l|}
\hline $\begin{array}{l}\text { Die neuen fiktiven Straßennamen und die } \\
\text { Namen der Stadtteile aus Rufnamen }\end{array}$ & $\begin{array}{l}\text { Rüfke, Kóbi, Iczig, Mojzes, Áron, Salamon, Jaj- } \\
\text { telesz, Jókelesz, Pinkelesz }\end{array}$ \\
\hline $\begin{array}{l}\text { Familiennamen lebendiger jüdischer Perso- } \\
\text { nen als neue Toponyme }\end{array}$ & $\begin{array}{l}\text { Wodjaner utca, Chorin tér, Rothschild tér, Szt. } \\
\text { Gelléri Mór hegy }\end{array}$ \\
\hline $\begin{array}{l}\text { Der Berufsname Schächter als Bestandteil } \\
\text { fiktiver Toponyme }\end{array}$ & Sakter tér, Sakter, Közsakterozó híd \\
\hline Namen des Prozesses als fiktive Toponyme & $\begin{array}{l}\text { Eszter híd, Esler sziget, Rituális fürdö, Rabbi } \\
\text { sziget, Cap Funták, Cap Horánszky }\end{array}$ \\
\hline $\begin{array}{l}\text { Stereotype als fiktive Toponyme: Essge- } \\
\text { wohnheiten }\end{array}$ & $\begin{array}{l}\text { Maczesz utca, Disznó tér, Liba hegy, Libamáj } \\
\text { utca }\end{array}$ \\
\hline $\begin{array}{l}\text { Stereotype als fiktive Toponyme: (wirtschaft- } \\
\text { liche) Tätigkeiten der Juden }\end{array}$ & $\begin{array}{l}\text { Fő Rebach tér, Hunczutka utca, Zsebmetszö } \\
\text { utca, Svindler utca, Alliancz híd }\end{array}$ \\
\hline $\begin{array}{l}\text { Motivation der Namengebung: ähnlicher } \\
\text { Klang }\end{array}$ & $\begin{array}{l}\text { Szt. Gelléri Mór hegy, Czepel sziget, Nyuzoda, } \\
\text { Csiszár fürdö, Mojzeum }\end{array}$ \\
\hline
\end{tabular}

Schließlich findet man auch im Titel der Stadtkarte interessante Elemente: „Die Ingenieure von Bolond Istók geben sie [die Karte] unter der Herrschaft von Tiszaeszlari Kogálman aus“. Kálmán Tisza war in diesem Zeitraum der ungarische Ministerpräsident, und er akzentuierte immer, dass die Juden unschuldig sind. Sein Name wurde aber verändert um zu suggerieren, dass er gute Beziehungen mit den Juden pflegt, und dass die Juden deswegen für unschuldig erklärt wurden, weil die Juden Tisza, die Regierung und das Gericht korrumpierten. Aus „Tisza“ konnten die Autoren leicht Tiszaeszlári machen, und die Leser verstanden vermutlich die Botschaft. Kogálman besteht aus den Elementen Kogál/Kagál und man. Das ungarische Wort „kagál“" stammt aus dem hebräischen Wort „Kahal“, das Religionsgemeinschaft heißt 
(Ujvári 1929: 446). Im „kagál“ vermuteten die ungarischen Antisemiten wieder eine geheime Organisation der Juden wodurch sie die Weltmacht ergreifen werden.

\section{Zusammenfassung}

Die oben aufgelisteten Beispiele illustrieren gut, dass die Namen - unabhängig davon, ob die Juden oder die Verteidiger der Juden sie bekamen - sehr effektive Waffen der zeitgenössischen Journalisten der analysierten Witzblätter waren. Mithilfe der Namen konnte man nicht nur die Juden identifizieren, sondern auch zahlreiche Stereotype wurden schnell mobilisiert, ohne dass man auf diese Stereotype und Vorurteile mit anderen Mitteln hinweisen sollte. In dieser Hinsicht sind die Namen auf Ungarisch und auf Deutsch genauso wirksam. Die erfundenen oder umformulierten Namen zeigen daneben auch die Kreativität der Autoren. Die Texte der antisemitischen Witzblätter kritisierten den Prozess der Namenmagyarisierung, die Charakterzüge der alten, heroischen Adeligen wurden mit den Eigenschaften der Juden verglichen, die Ergebnisse der Juden wurden auf dem Gebiet der Modernisierung Ungarns oder der ungarischen Wirtschaft erniedrigt.

Der Gattung Witzblatt gemäß übertrieben, aber die Autoren machten auf wahre Tendenzen aufmerksam, wiederspiegelten die Texte und Karikaturen die gesellschaftlichen Auseinandersetzungen, und dazu war es ein ausreichendes Mittel die zutreffende Namengebung der Figuren, Straßen, Stadtteile oder Gebäude.

Die Forschung wurde durch das Projekt EFOP-3.6.2-16-2017-00007 „Die Aspekte der Entwicklung einer intelligenten, nachhaltigen und inklusiven Gesellschaft: soziale, technologische, Innovationsnetze in der Beschäftigung und in der digitalen Wirtschaft" unterstützt. Das Projekt wird von der Europäischen Union finanziert, und vom Europäischen Sozialfonds und Ungarischen Staatshaushalt kofinanziert.

\section{Bibliographie}

Császtvay, T. 2009. Éjjeli lepkevadászat. Bordélyvilág a történeti Magyarországon [Nächtliche Jagd auf Schmetterlinge: Die Welt der Bordelle im historischen Ungarn]. Budapest: Osiris.

Farkas, T. 2002. Nyelvmüvelés és családnév-választás [Sprachpflege und die Wahl der Familiennamen]. In Éltetö anyanyelvünk. Irások Grétsy László 70. születésnapjára [Unsere lebendige Muttersprache. Festschrift für das 70. Geburtstag von László Grétsy], G. Balázs und A. Jászó und Á. Koltói (Hrsg.), 159-163. Budapest: Tinta Könyvkiadó.

Fischer, R. 1988. Entwicklungsstufen des Antisemitismus in Ungarn. 1867-1939: Die Zerstörung der magyarisch-jüdischen Symbiose. München: Oldenbourg.

Gottas, F. 1976. Ungarn im Zeitalter des Hochliberalismus: Studien zur Tisza-Ära, 1875-1890. Wien: Österreichische Akademie der Wissenschaften.

Karády, V. und I. Kozma. 2002. Név és nemzet: családnév-változtatás, névpolitika és nemzetiségi erőviszonyok Magyarországon a feudalizmustól a kommunizmusig [Name und Nation. Wechsel der Familienname, Namenpolitik und nationales Kräfteverhältnis von dem Feudalismus bis zum Kommunismus in Ungarn]. Budapest: Osiris. 
Kossuth, F. (Hrsg.). 1904. Kossuth Lajos iratai [Die Schriften von Lajos Kossuth]. Bd. 10, Budapest: Athenaeum.

Maitz, P. 2009. Névmagyarosítás és névideológia a dualizmus kori Magyarországon [Namenmagyarisierung und Namenideologie im Zeitalter des Dualismus in Ungarn]. In A családnév-változtatások történetei idöben, térben, társadalomban [Der Wechsel der Familiennamen in historischer Zeit, Raum und Gesellschaft], T. Farkas und I. Kozma (Hrsg.), 77-93. Budapest: Gondolat-Magyar Nyelvtudományi Társaság.

Nagy, I.G. 1992-1993. A névmagyarosítás történetéhez [Zur Geschichte der Namenmagyarisierung]. Baranya. Történelmi Közlemények 5-6: 63-77.

Tamás, Á. 2015. Namen der jüdischen Figuren in der zweiten Hälfte des 19. Jahrhunderts in österreichischen und ungarischen Witzblättern. In "Name and Naming" Conventional / Unconventional in Onomastics: Proceedings of the Third International Conference on Onomastics, O. Felecan (ed.), 365-375. Cluj-Napoca: Editura Mega, Argonaut.

Ujvári, P. 1929. Magyar zsidó lexikon [Ungarisches jüdisches Lexikon]. Budapest: Pallas. 